Int. J. Dev. Biol. 61: 763-772 (2017)

doi: $10.1387 / \mathrm{ijdb} .170139 \mathrm{zk}$

\title{
The opsin repertoire of the European lancelet: a window into light detection in a basal chordate
}

\author{
CHRYSOULA N. PANTZARTZII,\#, JIRI PERGNER ${ }^{2, \#,}$, IRYNA KOZMIKOVA ${ }^{2}$ and ZBYNEK KOZMIK*,1,2, \\ ${ }^{1}$ Laboratory of Eye Biology, Institute of Molecular Genetics of the Czech Academy of Sciences, Prague, Czech Republic, \\ Division BIOCEV, Vestec, Czech Republic and ' 2 Laboratory of Transcriptional Regulation, \\ Institute of Molecular Genetics of the ASCR, Prague, Czech Republic
}

\begin{abstract}
Light detection in animals is predominantly based on the photopigment composed of a protein moiety, the opsin, and the chromophore retinal. Animal opsins originated very early in metazoan evolution from within the G-Protein Coupled Receptor (GPCR) gene superfamily and diversified into several distinct branches prior to the cnidarian-bilaterian split. The origin of opsin diversity, opsin classification and interfamily relationships have been the matter of long-standing debate. Comparative studies of opsins from various Metazoa provide key insight into the evolutionary history of opsins and the visual perception in animals. Here, we have analyzed the genome assembly of the cephalochordate Branchiostoma lanceolatum, applying BLAST, gene prediction tools and manual curation in order to predict de novo its complete opsin repertoire. We investigated the structure of predicted opsin genes, encoded proteins, their phylogenetic placement, and expression. We identified a total of 22 opsin genes in B. lanceolatum, of which 21 are expressed and the remaining one appears to be a pseudogene. According to our phylogenetic analysis, representatives from the three major opsin groups, namely C-type, the R-type and the Group 4, can be identified in $B$. lanceolatum. Most of the $B$. lanceolatum opsins exhibit a stage-specific, but not a tissue-specific, expression pattern. The large number of opsins detected in $B$. lanceolatum, the observed similarities and differences in terms of sequence characteristics and expression patterns lead us to conclude that there may be a fine tuning in opsin utilization in order to facilitate visually-guided behavior of European amphioxus under various environmental settings.
\end{abstract}

KEY WORDS: Branchiostoma, amphioxus, opsin, expression

\section{Introduction}

Light sensing systems have evolved to be uniquely suited to the environment and behavior of any given species. Animals detect light using sensory cells known as photoreceptors, present in the eyes or, in case of extraocular photoreceptors, outside of the eyes. Although other systems of light detection exist in the animal kingdom, such as cryptochromes (Rivera et al., 2012) or LITE-1 (Gong et al., 2016), opsins are dominantly utilized as visual pigments among Metazoa. Opsins are seven transmembrane domain proteins that belong to the G-Protein Coupled Receptor (GPCR) superfamily and are canonically distinguished from other GPCRs by a highly conserved lysine in the seventh helix. The number of opsin genes differs significantly among species studied so far and does not generally correlate with the overall level of body plan sophistication. Cnidaria for example, despite having a relatively simple body plan and limited number of cell types, are known to possess a large number of opsins originating by species-specific gene duplications (Koyanagi et al., 2008; Kozmik et al., 2008; Suga et al., 2008). Opsin classification, interfamily relationships and evolution of animal vision have been the debate of numerous studies so far (D'Aniello et al., 2015; Feuda et al., 2012; Liegertova et al.,

Abbreviations used in this paper: CNS, Central Nervous System, GPCR, G-protein coupled receptor, TM, transmembrane.

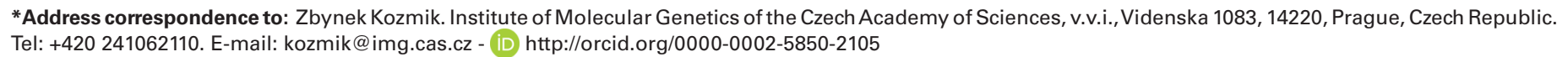

\#Note: These authors contributed equally.

Supplementary Material for this paper is available at: http://dx.doi.org/10.1387ijdb.170139zk

Submitted: 21 June, 2017; Accepted: 11 July, 2017.

ISSN: Online 1696-3547, Print 0214-6282

(C) 2017 UPV/EHU Press

Printed in Spain 
2015; Peirson et al., 2009; Plachetzki et al., 2007; Porter et al., 2012; Ramirez et al., 2016; Shichida and Matsuyama, 2009; Suga et al., 2008; Terakita, 2005). Opsins can be roughly clustered into four major groups, namely the ciliary opsins expressed in ciliary photoreceptors (Ctype), the rhabdomeric opsins expressed in rhabdomeric photoreceptors (R-type), the Group 4 opsins, and the Cnidarian opsins. A further subdivision of the first three groups into seven subfamilies was suggested (Lamb et al., 2007; Terakita, 2005), based on their sequence and on the type of the G-protein to which they are coupled: 1) the vertebrate visual and non-visual, 2) the encephalopsin/ TMT, 3) the Gq-coupled/melanopsin, 4) the neuropsin, 5) the Go-coupled, 6) the peropsin, and 7) the retinal photoisomerase subfamilies. Ramirez and colleagues have proposed that a repertoire of at least nine opsin paralogs was present in the bilaterian ancestor (Ramirez et al., 2016). In regard to the cnidarian opsins, there are conflicting results regarding their position in the phylogenetic tree (Feuda et al., 2012; Liegertova et al., 2015; Plachetzki et al., 2007; Porter et al., 2012; Suga et al., 2008). The function of most opsins consists of two steps: light absorption and G-protein activation in both visual and non-visual systems. Isomerization of the chromophore 11-cis-retinaldehyde to all-trans-retinaldehyde due to light absorption, changes the conformation of the opsin and triggers a signal transduction cascade, the type of which is dependent on the G-protein to which the opsin binds

Fig. 1. Molecular phylogenetic analysis of opsins by Maximum Likelihood method. The evolutionary history of opsin proteins was inferred by using the Maximum Likelihood method based on the Le_Gascuel_2008 model. The tree with the highest log likelihood is shown. Bootstrap values are shown (only values $>50$ ) either at the nodes or above the branches in the case of collapsed subgroups (e.g. neuropsin). A discrete Gamma distribution was used to model evolutionary rate differences among sites (2 categories). The tree is drawn to scale, with branch lengths measured in the number of substitutions per site. The analysis involved 787 amino acid sequences. There were a total of 419 positions in the final dataset (third cytoplasmic loop is excluded). Numbers in yellow boxes correspond to the bilaterian opsin paralogs identified by Ramirez et al., 2016.

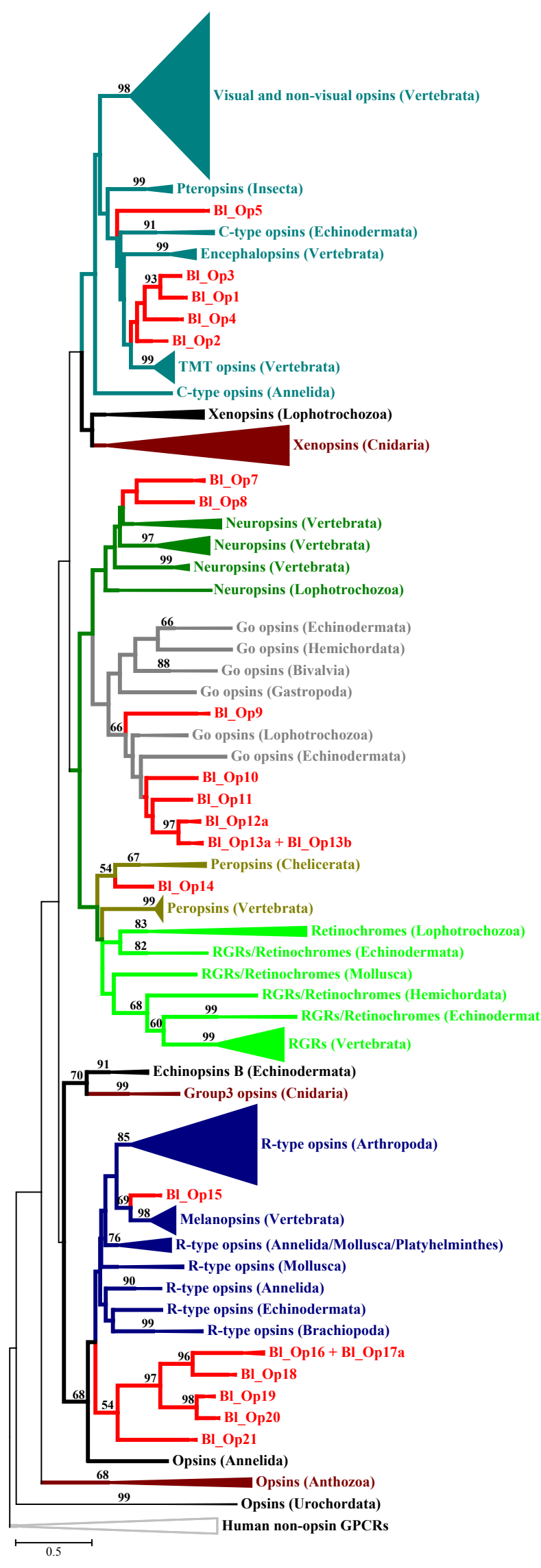

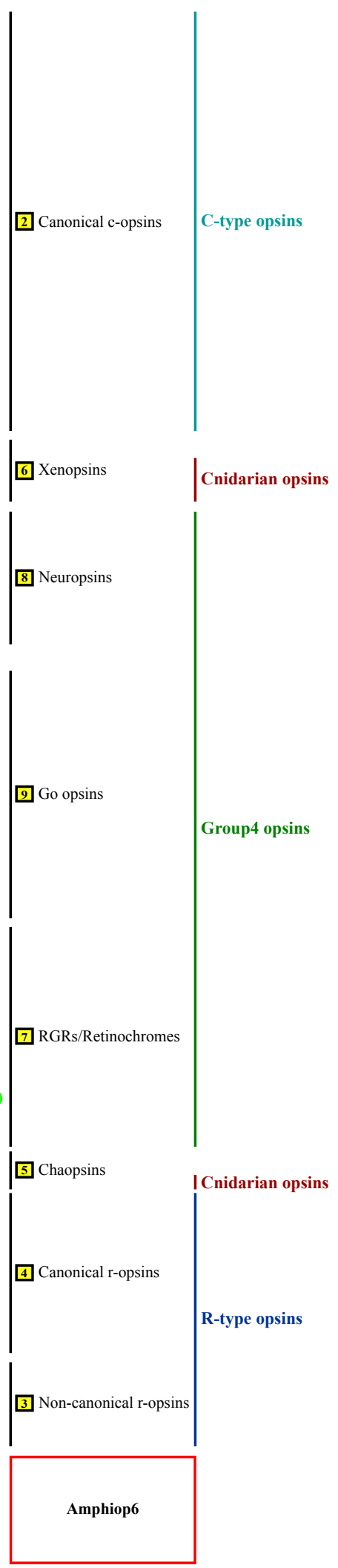

| Cnidarian opsins 
TABLE 1

NOMENCLATURE FOR BRANCHIOSTOMA OPSIN GENES

\begin{tabular}{|c|c|c|c|c|}
\hline Group & This study & $\begin{array}{c}\text { Branchiostoma floridae } \\
\text { models }\end{array}$ & $\begin{array}{l}\text { B. belcheri mRNA } \\
\text { sequences }\end{array}$ & References \\
\hline \multirow{4}{*}{ C-type opsins } & $\begin{array}{l}\text { BI_op1 (MF464463) } \\
\text { BI_op2 (MF464464) }\end{array}$ & $\begin{array}{l}124039 \\
205982\end{array}$ & AB050610 & c-opsin1 (Vopalensky et al., 2012), Amphiop5 (Koyanagi et al., 2002) \\
\hline & BI_op3 (MF464465) & 84894 & AB050609 & c-opsin3 (Vopalensky et al., 2012), Amphiop4 (Koyanagi et al., 2002) \\
\hline & BI_op4 (MF464466) & 70447 & & \\
\hline & BI_op5 (MF464467) & 84890 & & \\
\hline \multirow{3}{*}{ Neuropsins } & Not identified & 210643 & & \\
\hline & BI_op7 (MF464468) & 65045 & & \\
\hline & BI_op8 (MF464469) & 94083 & & \\
\hline \multirow{6}{*}{ Go opsins } & BI_op9 (MF464470) & 71561 & AB050607 & Amphiop2 (Koyanagi et al., 2002) \\
\hline & BI_op10 (MF464471) & 215180 & & \\
\hline & BI_op11 (MF464472) & 84844 & & \\
\hline & BI_op12a (MF464473) & 91094 & AB050606 & Amphiop1 (Koyanagi et al., 2002) \\
\hline & BI_op13a (MF464474) & 91095 & & \\
\hline & Bl_op13b (MF464475) & Not identified & & \\
\hline Peropsins & BI_op14 (MF464476) & 90832 & AB050608 & Amphiop3 (Koyanagi et al., 2002) \\
\hline Melanopsins & BI_op15 (MF464477) & 65960 & AB205400 & AmphMop (Koyanagi et al., 2005) \\
\hline \multirow{7}{*}{ Amphiop6 } & BI_op16 (MF464478) & 86640 & & \\
\hline & BI_op17a (MF464479) & 201585 & & \\
\hline & BI_op17b (MF464480) & Not identified & & \\
\hline & BI_op18 (MF464481) & Not identified & & \\
\hline & BI_op19 (MF464482) & 87094 & AB050611 & Amphiop6 (Koyanagi et al., 2002) \\
\hline & BI_op20 (MF464483) & 110003 & & \\
\hline & BI_op21 (MF464484) & 86195 & & \\
\hline
\end{tabular}

* Models from JGI, genome version v1.0 (http://genome.jgi.doe.gov/Brafl1/Brafl1.home.html)

(Koyanagi et al., 2008; Liegertova et al., 2015; Yarfitz and Hurley, 1994). The conserved lysine in the seventh helix is used to form a Schiff-base bond to the retinal chromophore (Bownds, 1967; Wang et al., 1980) and in its protonated form it is stabilized by a negatively charged amino acid, called a counterion, whose position varies among different opsin subfamilies (reviewed in Porter et al., 2012; Shichida and Matsuyama, 2009).

Comparative studies of opsins provide valuable insight not only into the origins of opsin diversity but also into the evolution of visual organs and light perception in animals. To help answer questions about vertebrate evolution at the key invertebrate chordate-vertebrate transition, such as "How did the vertebrate eye evolved?", one can examine opsins and visual organs of the extant and most basally divergent chordates, the cephalochordates. The subphylum Cephalochordata, a.k.a. amphioxus or lancelet, consists of approximately 29 extant species, with a worldwide distribution (Poss and Boschung, 1996). Amphioxus possesses four types of photoreceptive systems - the dorsal ocelli and the Joseph cells are rhabdomeric receptors, while the frontal eye and the lamellar body contain ciliary photoreceptors (Lacalli, 2004). Function of the first two receptors is still not clear, nevertheless, rhabdomeric receptors similar to Joseph cells are present in the cerebral eyes of tunicates (salps). Amphioxus frontal eye is considered homologous to the paired eyes of vertebrates, since its photoreceptors and pigment cells co-express a combination of transcription factors and opsins typical of the vertebrate eye photoreceptors (Vopalensky et al., 2012). The lamellar body, on the other hand, is proposed as the amphioxus homolog of the pineal gland (Lacalli, 2004). Seven mRNA (Amphiop1-Amphiop6 and AmphiMop) and 20 opsin genes have been previously identified in $B$. belcheriand $B$. floridae, respectively (Holland et al., 2008; Koyanagi et al., 2005; Koyanagi et al., 2002). There are representatives from the encephalopsin/TMT, neuropsin, Go, peropsin and melanopsin subfamilies; a divergent subfamily, Amphiop6 appears to be specifically duplicated in amphioxus (Holland et al., 2008). No homologs of the vertebrate visual/nonvisual opsin subfamily were detected (Holland et al., 2008). Studies on expression pattern and function of the Branchiostoma opsins are rather limited; the biochemical data support AmphiOp1 as a typical visual pigment and AmphiOp3 as a photoisomerase (Koyanagi et al., 2002), while the properties of the B. belcheri melanopsin homolog were found to resemble those of the visual opsins present in the intrinsically photosensitive rhabdomeric photoreceptor cells of vertebrates (Koyanagi et al., 2005). In agreement with their phylogenetic placement, antibody staining revealed specific expression of two ciliary-type opsins in the ciliary photoreceptor cells of the $B$. floridae frontal eye (Vopalensky et al., 2012). Here, we report for the first time the opsin repertoire of $B$. lanceolatum (Pallas, 1774) and provide information on the expression patterns of opsin genes across multiple tissues and developmental stages.

\section{Results}

\section{Identification, classification and genome organization of opsin genes in Branchiostoma lanceolatum}

To identify the opsin gene repertoire in $B$. lanceolatum we used the available genome assembly provided by the Branchiostoma lanceolatum genome consortium. Applying BLAST searches and de novo gene prediction we were able to identify 22 opsin genes in $B$. lanceolatum, out of which BI_op 17b is a putative non-functional gene (pseudogene), since it bears a stop codon in the first exon. Predicted transcripts and encoded proteins for newly characterized opsins in $B$. lanceolatum, details on gene organization and genomic location are provided in Supplementary file 1. In general, all predicted opsins have seven transmembrane helices and a lysine in the seventh helix. We did not detect any intronless opsins in B. lanceolatum.

The large scale phylogenetic relations of opsins has been the object of many studies (Albalat, 2012; Liegertova et al., 2015; Porter et al., 2012; Ramirez et al., 2016). We wanted to classify the $B$. lanceolatum opsins and investigate their distribution over the major opsin groups. We combined the available datasets from the most recent analyses and enriched them with the 21 newly 
identified homologs from $B$. lanceolatum; BI_Op17b was omitted, for being the product of a putative pseudogene. Two different alignments were used for our phylogenetic analyses, excluding (Fig. 1 and Supplementary Fig. S1) and including (Suppl. Fig. S2) the variable third cytoplasmic loop. In both datasets, the four traditional opsin groups, i.e. the C-type, the R-type, Group4 and the Cnidarian opsins, were recovered along with some other groups, albeit supported by low bootstrap values in many cases. Clustering of $B$. lanceolatum opsins is the same in both cases. Few differences were observed between the two datasets; two of the most striking are the relative positioning of R-type and Group4 opsins and the placement of the clade containing the CiNut homolog (Etani and Nishikata, 2002) (Fig. 1 and Supplementary Fig. S1 and Fig. S2). In Fig. 1, the major cnidarian group together with a small set of lophotrochozoan opsins (the Xenopsin according to Ramirez et al., 2016) cluster as a sister group to C-type opsins. Group4 is closer to C-type and the Xenopsin group, than the R-type group. Chaopsin (Ramirez et al., 2016), consisting of Echinopsin B (D'Aniello et al., 2015) and a small clade of cnidarian opsins namely Group3 (Mason et al., 2012), an anthozoan and a Ciona-specific clade are identified in our phylogenetic trees (Fig. 1 and Supplementary Fig. S1 and Fig. S2).

Members from almost all opsin subfamilies have been identified for B. lanceolatum (Fig. 1 and Suppl. Fig. S1 and Fig. S2). Five belong to the C-type (BI_Op1-5), two are in a clade sister to neuropsins (BI_Op7 and BI_Op8), six cluster with the Go opsins (Op9-Op13b), one with peropsins (Op14), one with melanopsins (BI_Op15) and six within the Amphiop6 clade (BI_Op16-BI_Op21). Based on sequence similarity and genomic location, BI_Op17b should be a member of the Amphiop6 group. No homologs of the vertebrate visual/non visual opsins have been detected. $B$. lanceolatum genes identified in this study, arranged in groups according to their phylogenetic placement, and their relation to previously identified homologs from two other Branchiostoma species are provided in Table 1. Next, we wanted to visualize how opsin genes are arranged in the genome of $B$. lanceolatum and whether there is some genetic linkage between opsin gene paralogs. The $B$. lanceolatum opsin-containing loci identified during our in silico analysis are depicted in Fig. 2. In particular, opsin genes are spread over 16 genomic regions (scaffolds) in $B$. lanceolatum, whereas in some cases, members of the same group are clustered in the same locus (scaffold), for example the Amphiop6 BI_op19 and Bl_op20 (Fig. 2).

Table 2 summarizes the residues found in the three putative counterion positions, as well as the tripeptide associated with the binding to G-proteins (Arendt etal., 2004; Marin etal., 2000). Aspartic acid is present at position 83 of not only the melanopsin ortholog (BI_Op15) but of most of the rest of the opsins. In almost all cases,
C-type opsins

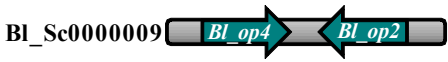

Group 4 opsins

(Neuropsins, Go-opsins, peropsins/RGR opsins)

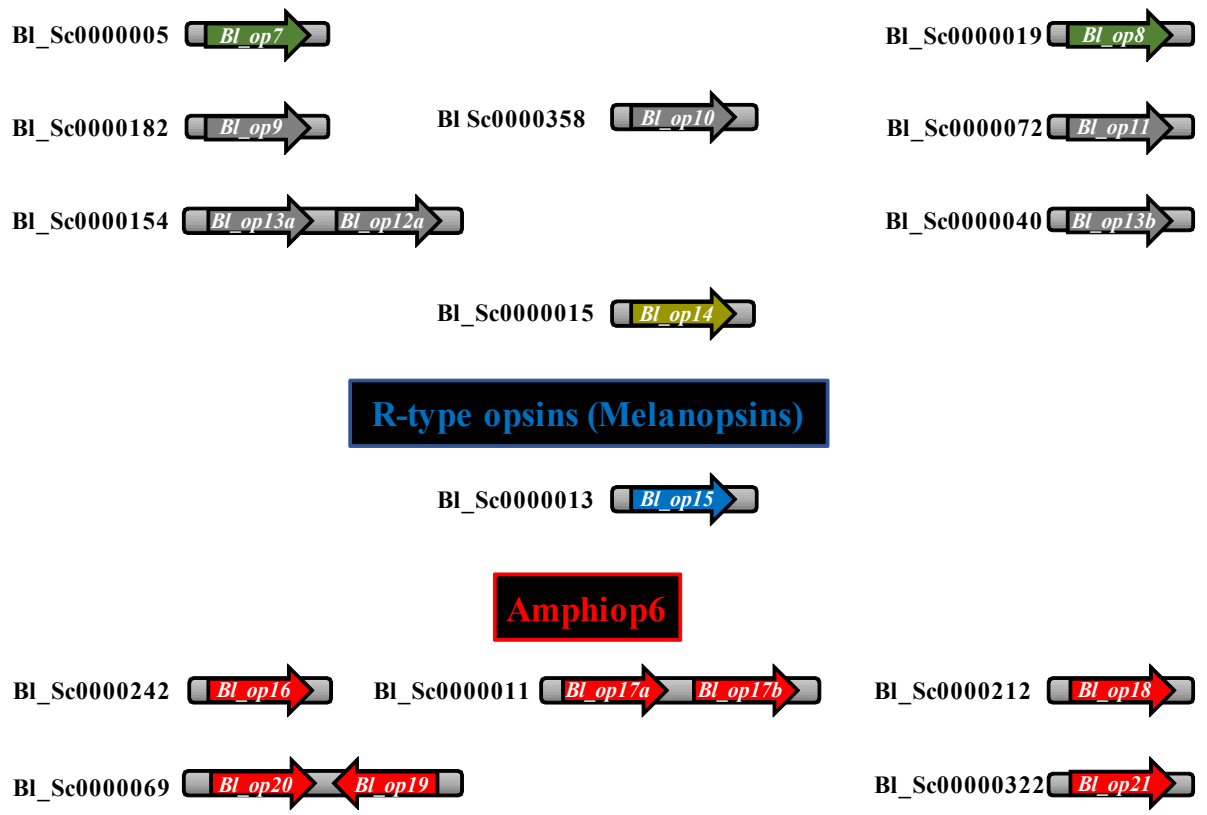

B1_Sc00000322 $\square$ Bl op21

Fig. 2. Opsin containing genomic loci from Branchiostoma lanceolatum. The genomic scaffolds from Branchiostoma lanceolatum (BI) containing opsin genes. Groups are colored based on their phylogenetic position (see Fig. 1).

position 113 is occupied by neutral or non-polar amino acids with the striking exception of BI_Op11, BI_Op19 andBI_Op20, for which a negatively charged amino acid is present, similarly to vertebrate visual and non-visual opsins. In fact, Bl_Op11 bears aspartic acid in both positions 83 and 113, but no negatively charged amino acid residue at position 181 . In the case of BI_Op5, a negatively charged amino acid is encountered only at position 83 . A really interesting case is that of $\mathrm{BI}$ OOp21, in the sense that no negative amino acid is present at any of the three putative counterion positions. All of the C-type opsins, except for BI_Op5, possess the N(N/S)Q tripeptide; the encountered motif in $\mathrm{BI}$ _Op5 is EKE. Melanopsins nicely bear the R-type tripeptide HPK (Arendt et al., 2004). This is not the case for BI_Op16-BI_Op22 (AmphiOp6); they may closely cluster with the R-type opsins but they only have the central proline, while the first position is occupied by a non-polar amino acid.

\section{B. Ianceolatum opsin gene expression patterns}

Opsin genes are used by many animals not only for visual, but also for non-visual tasks. Their tissue specificity thus can significantly vary. Recent analyses of opsin gene expression in Cnidaria or Arthropoda documented a wide range of tissues where opsins can be detected (Liegertova et al., 2015; Battelle et al., 2016). Our study examined the expression pattern of opsins across multiple developmental stages (Fig. $3 \mathrm{~A}$ and $\mathrm{B}$ ), as well as in multiple tissue types of $B$. lancelatum adult body (Fig. $3 C$ and D). To achieve this 
we performed quantitative real-time PCR (qRT-PCR). We scanned opsin expression in developmental stages starting from late neurula (N3), where photoreceptive $1^{\text {st }}$ Hesse cell is present, to adult, where frontal eye, rudiments of lamellar body, Joseph cells and series of dorsal ocelli along the neural tube are fully developed (Fig. 3A). All B. lanceolatum examined genes were detected at mRNA level, in at least one developmental stage (Fig. 3B and Supplementary Fig. S3A), with the sole exception of BI_op17b. This is in accordance with the in silico prediction of this gene as a pseudogene due to a premature stop codon at the beginning of the coding region. The neuropsin BI_op7 and the Amphiop6 BI_op16 show their highest expression in N3 neurula stage; in fact Bl_op7 is among the dominating opsins at this stage (Supplementary Fig. S3A). However, their expression is significantly reduced at later stages (Fig. 3B). Onset of several other opsin gene expression starts at L1 stage, in which frontal eye and lamellar body (ciliary photoreceptive organs) start to develop. The maximal expression of two Go opsins (BI_op9 and BI_op10) and one Amphiop6 opsin (BI_op20) was observed at this stage. We should point out that actin was used as a reference gene for normalization, a fact that could lead to a false underrepresentation of opsin genes in later developmental stages (L2/3 or adult), when compared to neurula stage, where lower total number of cells are present. Despite this fact, the majority of the opsins show most predominant expression

\section{TABLE 2}

\section{AMINO ACIDS OBSERVED AT PUTATIVE COUNTERION POSITIONS AND THE TRIPEPTIDE AT THE FORTH CYTOPLASMIC LOOP FOR B. LANCEOLATUM OPSINS}
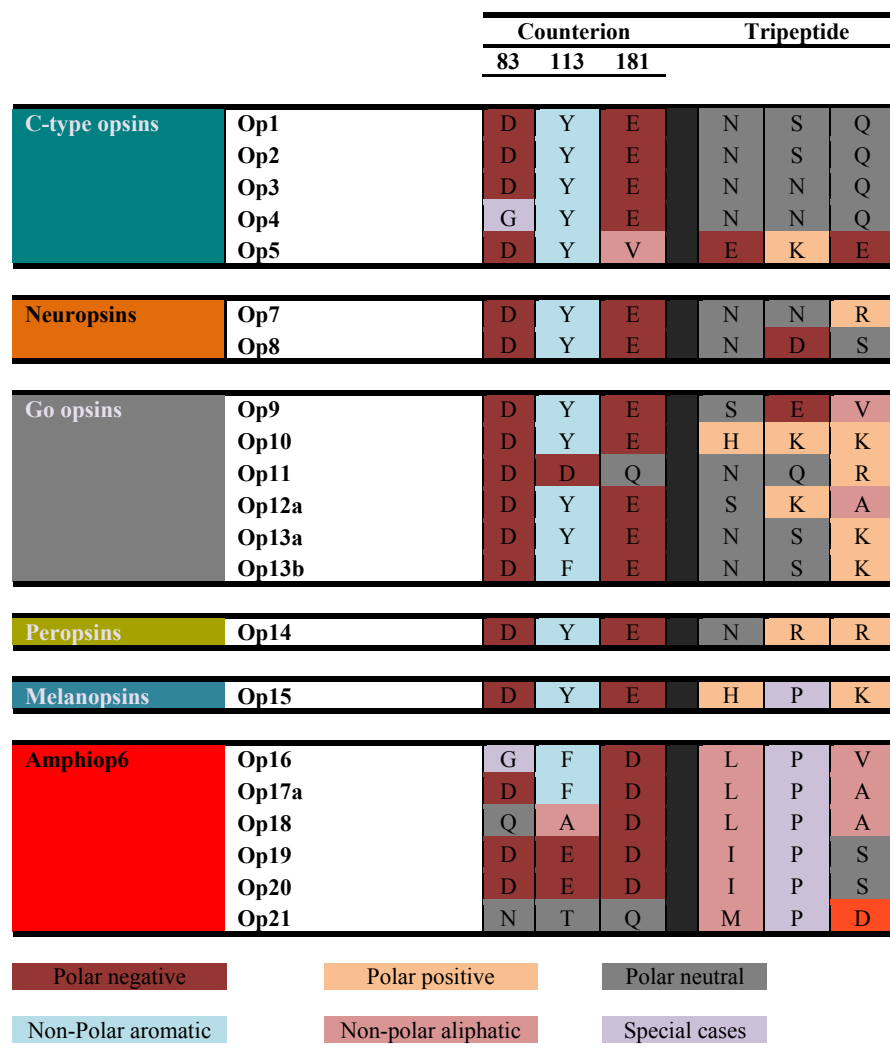

in L2/3 stage, where most of the known amphioxus photoreceptor organs - the frontal eye, the lamellar body and the Hesse cell - are differentiated. As expected from the ciliary nature of photoreceptor cells in the frontal eye and the lamellar body, all C-type opsins reach a peak in their expression in L2/3. On the other hand, B. lanceolatum opsins belonging to Go coupled opsin group show broad expression across various stages. Most of the Amphiop6 opsins show elevated expression in L2/3 stage, which is true also for the single peropsin (BI_op14). All of the 21 examined $B$. lanceolatum opsins could be detected in various adult tissues (Fig. $3 C$ and D). All $B$. lanceolatum opsins but $B$ I_op2, exhibit some specificity for either the cerebral vesicle (10 opsins - BI_op1, Bl_op4,BI_op5, Bl_op7,BI_op9,BI_op11,BI_op12a,BI_op13b,BI_op17a,BI_op20) or the neural tube (7 opsins - BI_op3, BI_op8, BI_op13a,BI_op14, BI_op15,BI_op18,BI_op21); on the contrary, BI_op5, BI_op17a, BI_op21 are expressed in both tissues (Fig. 3D and Supplement Fig. S3B). These findings show that most of the photoreceptive cells in amphioxus reside in the central nervous system, however, the cerebral vesicle and the neural tube probably exhibit a strong specialization for various photoreceptive tasks. Expression of one neuropsin (BI_op7) was documented in female gonads, whereas a Go and an Amphiop6 (BI_op13a and BI_op16, respectively) showed noticeable expression in male gonads (Fig. 3D). Interestingly, one of the C-type opsins (BI_op2) displays its highest level of expression in the most posterior part of the tail. Expression of four other opsins was significantly increased in tail. In contrast to analysis of opsin expression across various developmental stages (Fig. 3B), we were not able to observe any preference in tissue-specific usage of opsins belonging to different groups (Fig. 3D). The only exceptions were the peropsin (BI_op14) and the melanopsin (BI_op15), being highly expressed only in neural tube.

To investigate expression during larval development (prior to metamorphosis), we performed whole mount in situ hybridization. We analyzed expression of BI_op1, BI_op2, BI_op8, BI_op10, Bl_op11, Bl_op12a,BI_op14, BI_op15, BI_op17a, Bl_op19 and BI_op20. Of these, we have observed specific expression patterns only for BI_op11,Bl_op12 and BI_op15 (Fig. 4). BI_op11 is expressed in L1 stage in the area of developing preoral pit and $1^{\mathrm{st}}$ gill slit. In L2/3 the signal was detected in pharyngeal region and tail fin (Fig. 4 A,B). For Bl_op12a no specific signal was detected in L1 stage. In L2/3 stage the signal was detected in preoral pit, oral papilla, cells around mouth, $1^{\text {st }}$ gill slit and tail fin (Fig. 4C, D). In situ hybridization of BI_op15 in L1 and L2/3 stage identified specific expression in $1^{\text {st }}$ dorsal ocelli, in agreement with previous findings in B. belcheri (Koyanagi et al., 2005).

In summary, our analysis confirms expression of all but one $B$. lanceolatum opsin genes and documents their stage and/or tissue specificity.

\section{Discussion}

We sought to characterize the opsin gene family in the genome assembly of the cephalochordate Branchiostoma lanceolatum and study the expression patterns of opsin genes from this species in different tissue types and across various developmental stages. We identified a total of 22 opsin genes in $B$. lanceolatum, one of which is a putative pseudogene. There is less than $20 \%$ amino acid similarity between vertebrate opsin subfamilies but more than $40 \%$ among members of a single family (Peirson et al., 2009; Shichida 
and Matsuyama, 2009; Terakita, 2005). Given these low similarity levels, de novoprediction of opsin genes could be largely hampered. Therefore, BLAST results should be carefully filtered and used in combination with synteny analyses since true positive results could be obscured by low similarity scores. In addition, manual curation of the genome assembly was needed in some cases.

Discrepancies between different phylogenetic studies have been noted before and could be attributed to the dataset used, the alignment method, the substitution model, and the tree constructing method applied. In our study, we show how sensitive the outcome of the phylogenetic analysis can be to the exclusion or inclusion of the highly variable third cytoplasmic loop (Fig. 1 and Supplementary Fig. S2, respectively). Among the observed differences, two of the most striking are the relative positioning of R-type and Group4 opsins and the placement of the Urochordate opsins clade containing the CiNut homolog (Etani and Nishikata, 2002), for which inconsistencies were previously observed (Albalat, 2012; Porter et al., 2012; Ramirez et al., 2016). A new clade - "bathyopsin" - was recently introduced (Ramirez et al., 2016), consisting of one brachiopod and three echinoderm opsins. We excluded these sequences from our analysis either due to an incorrect number of transmembrane domains, based on TOPCONS and HMMTOP predictions (4 TM in the case of Strongylocentrotus purpuratus and 8 in the case of Lingula anatina) or because of their extremely small size (as in the case of Eucidaris tribuloides). It is obvious that there are still obstacles to reconstructing the complete evolutionary history of opsins. Sampling from specific taxonomic groups is still poor, mainly due to lack of data at the level of whole genome and missing functional data that could greatly facilitate the opsin classification. Collectively, our phylogenetic analysis ascribes $B$.
A

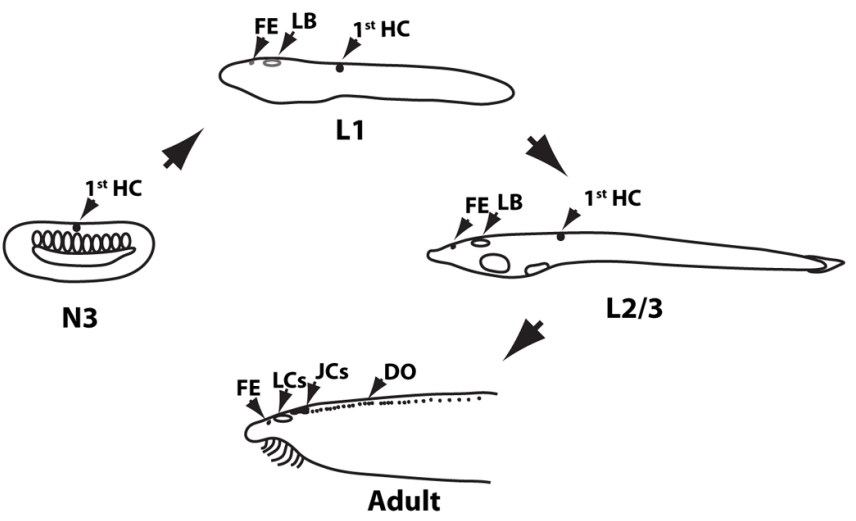

B

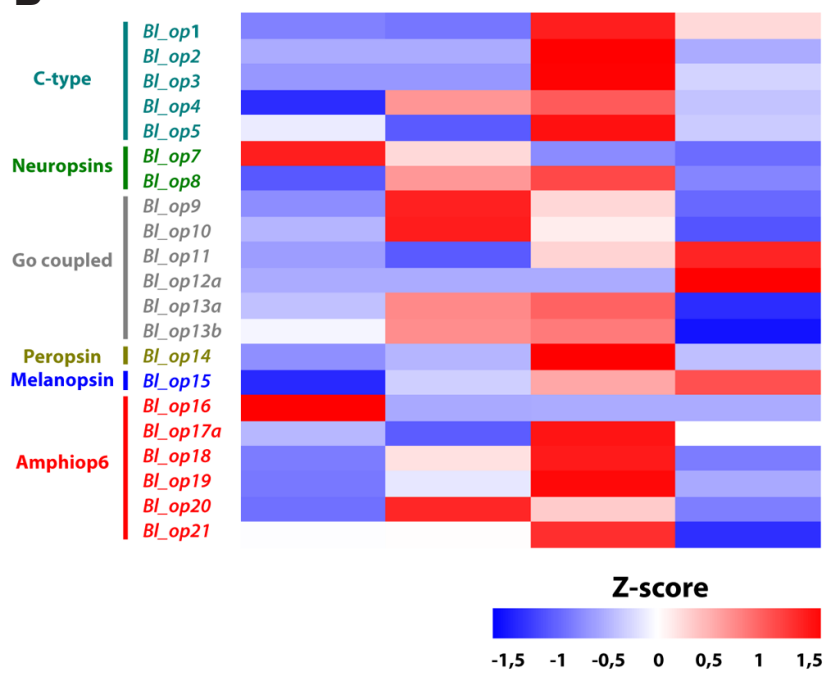

C

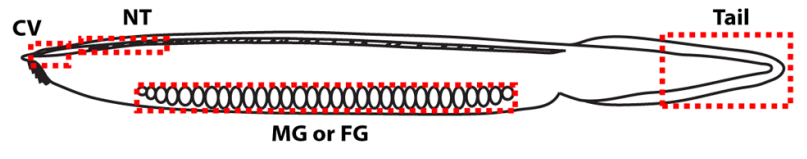

D
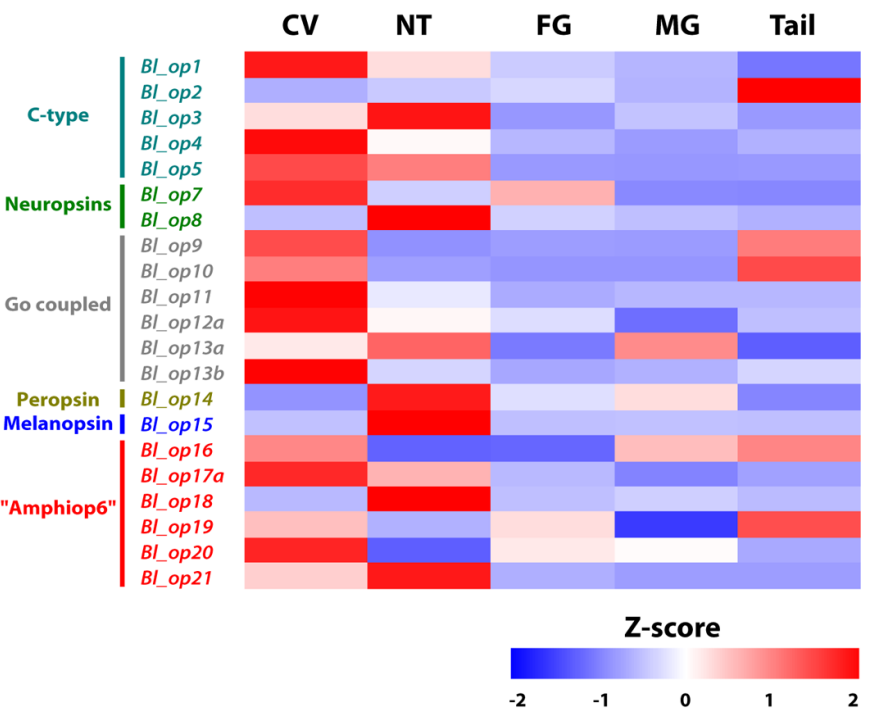

Fig. 3. mRNA expression levels of individual B. lanceolatum opsins across different developmental stages and various tissues of the adult body. (A) Schematic drawing of developmental stages (N3, L1, L2/3, adult), in which detection of opsin genes expression was performed. Staging was determined according to Hirakow and Kajita (1994) (see Materials and Methods). $1^{\text {st }}$ HC: $1^{\text {st }}$ Hesse cell, FE: frontal eye, LB: lamellar body, DO: dorsal ocelli, JCs: Joseph cells, LCs: lamellate cells (rudiment of larval lamellar body). (B) Heat map displaying expression of opsin genes across different developmental stages. Each row represents particular opsin gene expression in various developmental stages. (C) Schematic drawing of amphioxus adult body parts in which detection of opsin genes expression was performed. CV: cerebral vesicle, NT: the most anterior third of neural tube including Joseph cells and dense clusters of dorsal ocelli, FG: female gonads, MG: male gonads, Tail: most posterior part of adult tail without dorsal ocelli. (D) Heat map displaying expression of opsin genes in various parts of B. lanceolatum adult body. Opsin expression was detected by qRT-PCR and normalized to expression of actin. Each row represents particular opsin gene expression in various parts of amphioxus adult body. Blue color represents expression below row average, white color represents average row expression, red color expression above row average. 

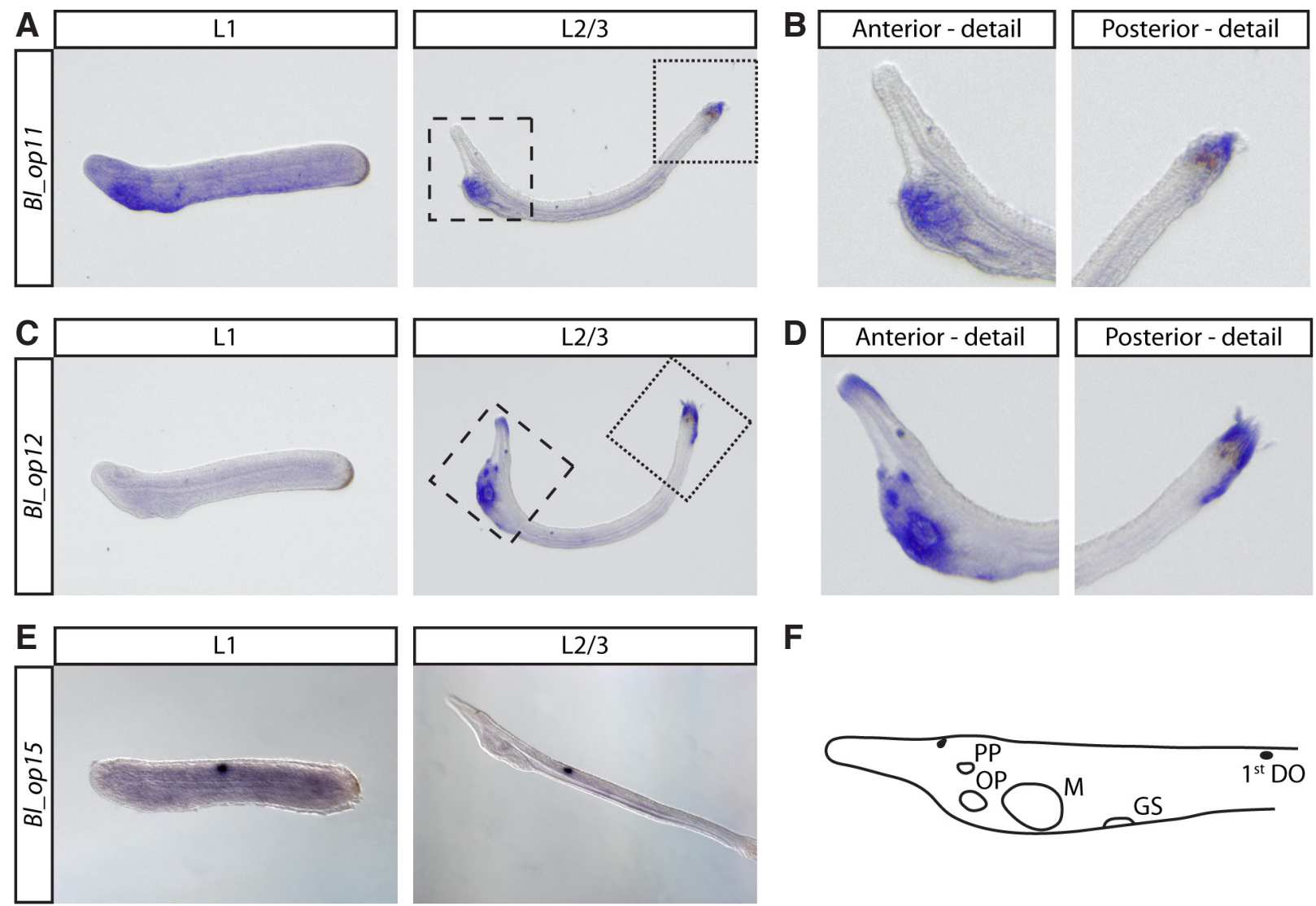

Fig. 4. In situ hybridization analysis of B. lanceolatum BI_op11, BI_op12a and BI_op15 in developing larvae (L1 and L2/3 stages). (A) Expression of $\mathrm{BI}$.op11. In stage $\mathrm{L} 1$ the signal was detected in the area of developing preoral pit and $1^{\text {st }}$ gill slit. In $L 2 / 3$ the signal was detected in pharyngeal region and tail fin. (B) Detail of anterior and posterior region of the larvae (dashed and dotted framed regions in (A)). (C) Expression of BI_op12a. In L1 stage no specific signal was detected. In L2/3 the signal was detected in preoral pit, oral papilla, cells around mouth, $1^{\text {st }}$ gill slit and tail fin. (D) Detail of anterior and posterior region of the larvae (dashed and dotted framed regions shown in (C)). (E) Expression of BI_op15. Specific signal was detected in 1 st dorsal ocelli. (F) Scheme of anterior part of L2/3 larvae with marked landmarks. PP, preoral pit; OP, oral papilla; M, mouth; GS, gill slit; $1^{\text {st }}$ DO, first dorsal ocelli.

lanceolatum opsins in the three of the four traditionally recognized opsin lineages, in particular C-type, R-type and Group4 (neuropsins, RGRs and peropsins), with no differences between the two sequence datasets used in this study. The number of genes in each subfamily, including a putative pseudogene, varies from one (melanopsins and peropsins) to a maximum of seven (Amphiop6). The expansion of three opsin subfamilies in the cephalochordate lineage (Amphiop6, C-type and Go-opsins) was previously (Holland et al., 2008) correlated to the diversity of photoreceptor organs in amphioxus, including both ciliary and rhabdomeric photoreceptors (Lacalli, 2004). The large size of the Go group suggests extensive redundancy and/or could be an indication of possible fine tuning of molecular properties among these opsins in order to achieve distinct photoreceptive properties.

In general, $B$. lanceolatum Go opsins exhibit a preferential expression in either the cerebral vesicle or the neural tube (parts of the Central Nervous System - CNS), with two interesting exceptions: BI_op13ain male gonads and BI_op9-op10 in tail. A CNS-specific pattern is observed for the C-type opsins as well, with BI_op2, however, being expressed almost solely in the tail. Similar is the expression in the horseshoe crab Limulus polyphemus, where various opsins have been detected in the tail (Battelle et al., 2016), proposed to be a circadian photoreceptor organ. Photoreceptors located in the tail mediate light avoidance in larval lampreys (Binder and McDonald, 2008). Light sensitivity in the tail of amphioxus has been reported as early as 1908 (Parker, 1908), yet no study so far has documented any photoreceptor cells there. In regard to opsin expression in gonads, it has also been observed in the gonads of the cnidarian species Cladonema radiatum (Suga et al., 2008) and Tripedalia cystophora (Liegertova et al., 2015), and the oyster Crassostrea gigas (Porath-Krause et al., 2016). The role in opsin-mediated light-controlled release of gametes was proposed (Liegertova et al., 2015; Suga et al., 2008). In agreement with data regarding the $B$. belcheri melanopsin (Koyanagi et al., 2005), an orthologous BI_op15 is expressed in the neural tube where rhabdomeric dorsal ocelli are located. Nevertheless, it is not the unique opsin expressed in this tissue. The $B$. lanceolatum peropsin (BI_op14), two C-type opsins, namely BI_op3 (ortholog of B. belcheri Amphiop4) and Bl_op5 (not previously analyzed in $B$. belcheri), neuropsin (BI_op8) and two Amphiop6 opsins (BI_op18 and BI_op21) were all found to be expressed in the neural tube of adult $B$. lanceolatum.

Examination of amino acid residues at key positions (namely, the counterion and the tripeptide on the fourth cytoplasmic loop) may provide insight into molecular function of individual opsins. Nevertheless, there are instances of a non-typical pattern in re- 
gard to these positions (Plachetzki et al., 2007). The presence of negative residues at position 113, typical for vertebrate opsins, is also evident in three Branchiostoma (this study) as well as in four Tripedalia (Liegertova et al., 2015) opsins. Among them is BI_Op11, which further lacks a negative residue at position 181, thus raising questions about its role and expression domain. This opsin could also provide some insight in regard to the timepoint that counterion replacement occurred during the molecular evolution of vertebrate opsins (Terakita et al., 2004). Slightly differentiated from the rest of C-type opsins is BI_Op5, for which the sole negatively charged residue is located at position 83. Another thing that should be highlighted is the absence of a negatively charged residue in any of the three putative counterion positions of the Amphiop6 BI_Op21. The type of signaling cascade activated by various opsins is summarized by Porter et al., 2012 and an in silico attempt has been made to correlate the motif present in the $\mathrm{N}$-terminal of the fourth cytoplasmic loop (a.k.a. the tripeptide) with the target G-proteins. As shown before, the NKQ motif of the rhodopsin holds an important role in the activation of the G-protein transducin (Marin et al., 2000). In regard to this position, some of our results are rather conventional, but some should attract more attention in the future. For example, BI_Op5 again stands out from the rest of the C-type opsins, since it contains the rather unique EKE motif. Amphiop6 genes possess only the central proline of the R-opsin HPK fingerprint, therefore their ability to couple to any downstream phototransduction cascades remains an open question. Apart from the conserved proline, there is no clear pattern for the tripeptide in Amphiop6 subgroup, except for the fact that a non-polar aliphatic residue occupies the first position. This group clusters closely with Gq opsins, however its members also differ in regard to motifs characteristic of Gq opsins, necessary for structural integrity maintenance and binding to the chromophore (Porath-Krause et al., 2016).

In summary, our genome-wide analysis identifies the complement of opsin genes in $B$. lanceolatum, confirms expression of all but one genes and documents their stage and/or tissue specificity. Studies of opsin diversity can offer clues to how separate lineages of animals have repurposed different opsin paralogs for different light-detecting functions. To gain a deeper insight into the function of amphioxus photoreceptive organs, more detailed expression analysis of individual opsins (e.g. by in situ hybridization or immunohistochemistry staining) in conjunction with light-mediated behavioral tests of animals is of key importance. In addition, studies aimed to dissect the biochemical properties of individual amphioxus opsins and the nature of the downstream phototransduction cascade are highly warranted. Such further studies may provide evidence for fine tuning of molecular properties within the pool of available opsins that were necessary to adapt visually guided behavior of amphioxus to changing habitats.

\section{Materials and Methods}

\section{Genome assembly and de novo gene prediction}

$B$. lanceolatumopsin genomic loci were detected through tBLASTn searches against the European amphioxus (v. Bl71nemh 20/11/13) Assembly (BraLan2). The previously characterized (Holland et al., 2008; Koyanagi et al., 2005; Koyanagi et al., 2002) Branchiostoma opsin homologs were used as queries. Additional BLAST searches were performed using various visual and non-visual homologs from vertebrate and protostome species. De novo prediction of $B$. lanceolatum opsin genes was accomplished combining results from Genscan (Burge and Karlin, 1997) and exon-intron borders predictions by SpliceView (Rogozin and Milanesi, 1997). In the case of discrepancies between database gene models and our in silico analysis, PCR amplification of the "suspicious" regions was performed, followed by cloning and sequencing (see paragraph "Cloning and Sequencing of Opsin Gene Fragments/Transcripts").

\section{Prediction of membrane protein topology and functional domains}

Newly identified $B$. lanceolatumopsin homologs were run in ScanProsite (de Castro et al., 2006), in order to identify protein family domains/motifs, more specifically "the G Protein Coupled Receptor (GPCR) signature/ profile" and the "Visual pigments (opsins) retinal binding site". In addition, TOPCONS (Tsirigos et al., 2015) and CCTOP (Dobson et al., 2015) were used to detect the protein topology in general and the exact position of the seven transmembrane (TM) helices, characteristic of the GPCRs in particular. In order for the proteins to be considered reliable opsin homologs they had to meet the following three criteria: 1) exhibit similarity to known opsins, 2) bear seven TM domains and 3) possess a lysine residue at the seventh TM domain.

\section{Sequence collection, alignments and phylogenetic analysis}

Given the fact that the datasets used in the most recent large scale analyses (D'Aniello et al., 2015; Liegertova et al., 2015; Porter et al., 2012; Ramirez et al., 2016) had included significant number of opsins representative of a large number of taxonomic groups, we first tried to combine the available datasets from them. Sequences of poor quality (large gaps, missing the K296, not bearing all seven TM domains) were omitted, predicted de novo or replaced by orthologs from relative species. We then enriched this dataset with the 21 newly identified homologs from $B$. lanceolatum.

Multiple sequence alignments were produced with the Clustal algorithm, incorporated in MEGA v7 (Kumar et al., 2016), and PROMALS3D (Pei et al., 2008). The latter constructs alignments for multiple protein sequences and/or structures using information from sequence database searches, secondary structure prediction, available homologs with 3D structures and user-defined constraints (Pei et al., 2008), therefore, it should be more reliable in the case of such a diverse group as opsins. Ambiguously aligned regions of the sequences, i.e. parts of the $\mathrm{N}$-terminal and $\mathrm{C}$-terminal ends were trimmed off in the MEGA7 alignment editor, leaving only the TM domains and the connecting extra-cellular and cytoplasmic loops. TOPCONS and CCTOP predictions were taken into consideration during the trimming process. We then created another subset in which the third cytoplasmic loop was removed from the alignment; the size of this loop is rather conserved within members of the same subfamily but it is highly variable between different families; it can fluctuate from 18aa in vertebrate RGRs, to 20aa in molluscan retinochromes, 30aa in human short-wave opsins and up to 73aa in echinopsins B. High degree of sequence dissimilarity is also observed, which renders alignment rather problematic.

Maximum Likelihood (ML) trees were constructed for both datasets. LG, gamma distributed (Le and Gascuel, 2008) was indicated as the best substitution model by the "Find Best DNA/Protein Models" tool incorporated in MEGA7 (Kumar et al., 2016). ML trees were built in MEGA7 and tree topologies were evaluated with the bootstrap test (100 pseudoreplicates). B. lanceolatum sequences are included in Supplementary file 1, accession numbers (ACs) for the rest of the protein sequences (or genomic scaffold in case of newly predicted sequences) used in the phylogenetic analysis are included in Supplementary Fig. 1. All trees were rooted with the 22 human non-opsin GPCRs used in previous studies as well (Liegertova et al., 2015).

\section{Animal collection}

B. lanceolatumadult animals were collected in Argeles-sur-Mer (France) and preserved in the lab in a day/night cycle of $14 \mathrm{~h} / 10 \mathrm{~h}$ until spawning, which was induced by a shift in temperature (Fuentes et al., 2007). Staging of all collected embryos was performed according to Hirakow and Kajita (1994). Specimens from late neurula (N3), larvae (L1-L2/3) and adult stage were collected and frozen in RNAlater® Stabilization Solution (ThermoFisher 
Scientific), under light conditions. In N3, $1^{\text {st }}$ Hesse cell is present, in L1 the developing frontal eye and lamellar body are present, in L2/3 the $1^{\text {st }}$ gill slit and the mouth are open, frontal eye and lamellar body have differentiated. In adult, frontal eye, rudiments of lamellar body, Joseph cells and series of dorsal ocelli are present.

B. lanceolatum adults were anesthetized with Tricaine methane sulfonate and dissected in order to obtain the required tissue types, specifically the cerebral vesicle, the most anterior third of neural tube including Joseph cells and dense clusters of dorsal ocelli, the female and the male gonads and finally the most posterior part of tail without dorsal ocelli. Tissues were stored in RNAlater® Stabilization Solution (ThermoFisher Scientific) and frozen.

\section{RNA isolation / cDNA preparation}

Total RNA was isolated from $B$. lanceolatum embryos or adult tissues stored in RNAlater ${ }^{\circledR}$ Stabilization Solution using the Trizol reagent (Ambion). To avoid genomic DNA contamination, isolated RNA was treated with DNasel and purified on RNeasy Mini Kit (Qiagen) column. Random-primed cDNA was prepared from 250ng or $150 \mathrm{ng}$ of RNA (for analysis of the different developmental stages or adult tissue types, respectively) in a $20 \mu$ l reaction using SuperScript VILO cDNA Synthesis kit (Invitrogen).

\section{Cloning and sequencing of opsin gene fragments/transcripts}

For validation of the in silico predicted gene models, cloning of opsin gene fragments and complete transcripts from $B$. lanceolatum was performed. Primers were designed in Primer3 software (Supplementary Table 1). PCR was performed on cDNA from neurula N3, larvae $L 2 / 3$ or adult stages, using DreamTaq polymerase (ThermoFisher Scientific) according to the manufacturer's protocol. Amplified fragments were blunted and cloned into $\mathrm{PCR}^{\mathrm{TM}}$-Blunt II-TOPO ${ }^{\circledR}$ (ThermoFisher Scientific). Plasmid DNA was isolated using GeneJET Plasmid Miniprep Kit (ThemoFisher Scientific). Sequencing was performed by the standard Sanger sequencing procedure (GATC Biotech).

\section{qRT-PCR}

Primers were designed in Primer3 software (Supplementary Table 1). The qRT-PCR was performed in the LightCycler 2.0 System using the LightCycler® 480 DNA SYBR Green I Master kit (Roche Diagnostics, Germany), according to the manufacturer's standard protocol. For each cDNA sample, both target and housekeeping genes were measured under the same conditions. Results were analyzed using the LightCycler software. Crossing point values $(\mathrm{Cp})$ were determined as an average of triplicates for each gene and normalized by $\mathrm{Cp}$ values of the housekeeping gene (actin). Results were analyzed in R software and plotted in the form of a Z-score heat map.

\section{Whole-mount in situ hybridization in amphioxus embryos}

Whole-mount in situ hybridization with digoxigenin-labeled RNA probes was done as previously described (Kozmikova et al., 2013). For better interpretation of the signal, in situ hybridization with Vector Blue substrate was performed in some cases, followed by subsequent confocal microscopic analysis. Primers used to generate probes are summarized in Supplementary Table 1.

\section{Acknowledgments}

This work was funded by the Czech Science Foundation (GACR, 17-15374S); Ministry of Education, Youth and Sports (LO1220 CZOPENSCREEN, LQ1604 NPU II, BIOCEV-CZ.1.05/1.1.00/02.0109) and the Institute of Molecular Genetics institutional support (RVO 6878050). This work was supported by the Branchiostoma lanceolatum genome consortium that provided access to the $\mathrm{B}$. lanceolatum genome sequence.

\section{References}

ALBALAT R (2012). Evolution of the genetic machinery of the visual cycle: a novelty of the vertebrate eye? Mol Biol Evol 29: 1461-1469.
ARENDT D, TESSMAR-RAIBLE K, SNYMAN H, DORRESTEIJN A W, WITTBRODT $\mathrm{J}$ (2004). Ciliary photoreceptors with a vertebrate-type opsin in an invertebrate brain. Science 306: 869-871.

BATTELLE B A, RYAN J F, KEMPLER K E, SARAF S R, MARTEN C E, WARREN W C, MINX P J, MONTAGUE M J, GREEN P J, SCHMIDT S A et al., (2016). Opsin repertoire and expression patterns in horseshoe crabs: evidence from the genome of Limulus polyphemus(Arthropoda:Chelicerata). Genome BiolEvol8: 1571-1589.

BINDER T R, MCDONALD D G (2008). The role of dermal photoreceptors during the sea lamprey (Petromyzon marinus) spawning migration. J Comp Physiol A Neuroethol Sens Neural Behav Physiol 194: 921-928.

BOWNDS D (1967). Site of attachment of retinal in rhodopsin. Nature216: 1178-1181.

BURGE C, KARLIN S (1997). Prediction of complete gene structures in human genomic DNA. J Mol Biol 268: 78-94

D'ANIELLOS, DELROISSE J, VALERO-GRACIAA, LOWE EK, BYRNE M, CANNON $\mathrm{J}$ T, HALANYCH K M, ELPHICK M R, MALLEFET J, KAUL-STREHLOW $S$ et al., (2015). Opsin evolution in the Ambulacraria. Mar Genomics 24, Part 2: 177-183.

DE CASTRO E, SIGRIST C J A, GATTIKER A, BULLIARD V, LANGENDIJK-GENEVAUX P S, GASTEIGER E, BAIROCHA, HULO N (2006). ScanProsite: detection of PROSITE signature matches and ProRule-associated functional and structural residues in proteins. Nucleic Acids Res 34: W362-W365.

DOBSON L, REMENYI I, TUSNADY G E (2015). CCTOP: a Consensus Constrained TOPology prediction web server. Nucleic Acids Res 43: W408-412.

ETANI K, NISHIKATA T (2002). Novel G-protein-coupled receptor gene expressed specifically in the entire neural tube of the ascidian Ciona intestinalis. Dev Genes Evol 212: 447-451

FEUDA R, HAMILTON S C, MCINERNEY J O, PISANI D (2012). Metazoan opsin evolution reveals a simple route to animal vision. Proc Natl Acad Sci USA 109: 18868-18872.

FUENTES M, BENITO E, BERTRAND S, PARIS M, MIGNARDOT A, GODOY L, JIMENEZ-DELGADO S, OLIVERI D, CANDIANI S, HIRSINGER E et al., (2007). Insights into spawning behavior and development of the European amphioxus (Branchiostoma lanceolatum). J Exp Zool B Mol Dev Evol 308: 484-493.

GONG J, YUAN Y, WARD A, KANG L, ZHANG B, WU Z, PENG J, FENG Z, LIU J, XU $X Z$ (2016). The $C$. elegans Taste Receptor Homolog LITE-1 Is a Photoreceptor. Cell 167: 1252-1263 e1210.

HIRAKOW R, KAJITA N (1994). Electron microscopic study of the development of amphioxus, Branchiostoma belcheri tsingtauense: the neurula and larva. Kaibogaku Zasshi 69: 1-13.

HOLLAND LZ, ALBALATR, AZUMIK, BENITO-GUTIERREZE, BLOWMJ, BRONNERFRASER M, BRUNET F, BUTTS T, CANDIANI S, DISHAW L J et al., (2008). The amphioxus genome illuminates vertebrate origins and cephalochordate biology. Genome Res 18: 1100-1111.

KOYANAGI M, KUBOKAWA K, TSUKAMOTO H, SHICHIDA Y, TERAKITAA (2005). Cephalochordate melanopsin: evolutionary linkage between invertebrate visual cells and vertebrate photosensitive retinal ganglion cells. Curr Biol 15: 1065-1069.

KOYANAGI M, TAKANO K, TSUKAMOTO H, OHTSU K, TOKUNAGA F, TERAKITA A (2008). Jellyfish vision starts with cAMP signaling mediated by opsin-G(s) cascade. Proc Natl Acad Sci USA 105: 15576-15580.

KOYANAGI M, TERAKITAA, KUBOKAWAK, SHICHIDAY (2002). Amphioxus homologs of Go-coupled rhodopsin and peropsin having 11-cis- and all-trans-retinals as their chromophores. FEBS Lett 531: 525-528.

KOZMIK Z, RUZICKOVA J, JONASOVA K, MATSUMOTO Y, VOPALENSKY P, KOZMIKOVA I, STRNAD H, KAWAMURA S, PIATIGORSKY J, PACES V et al., (2008). Assembly of the cnidarian camera-type eye from vertebrate-like components. Proc Natl Acad Sci USA 105: 8989-8993.

KOZMIKOVA I, CANDIANI S, FABIAN P, GURSKA D, KOZMIK Z (2013). Essential role of Bmp signaling and its positive feedback loop in the early cell fate evolution of chordates. Dev Biol 382: 538-554.

KUMAR S, STECHER G, TAMURA K (2016). MEGA7: Molecular Evolutionary Genetics Analysis Version 7.0 for Bigger Datasets. Mol Biol Evol 33: 1870-1874.

LACALLI T C (2004). Sensory systems in amphioxus: a window on the ancestral chordate condition. Brain Behav Evol 64: 148-162.

LAMB T D, COLLIN S P, PUGH E N, JR. (2007). Evolution of the vertebrate eye: opsins, photoreceptors, retina and eye cup. Nat Rev Neurosci 8: 960-976.

LE S Q, GASCUEL O (2008). An improved general amino acid replacement matrix. 


\section{C.N. Pantzartzi et al.}

Mol Biol Evol 25: 1307-1320.

LIEGERTOVAM, PERGNER J, KOZMIKOVAI, FABIAN P, POMBINHOAR, STRNAD H, PACES J, VLCEK C, BARTUNEK P, KOZMIK Z (2015). Cubozoan genome illuminates functional diversification of opsins and photoreceptor evolution. Sci Rep 5: 11885

MARIN E P, KRISHNAA G, ZVYAGATA, ISELE J, SIEBERT F, SAKMAR T P (2000). The amino terminus of the fourth cytoplasmic loop of rhodopsin modulates rhodopsin-transducin interaction. J Biol Chem 275: 1930-1936.

MASON B, SCHMALE M, GIBBS P, MILLER M W, WANG Q, LEVAY K, SHESTOPALOV V, SLEPAK V Z (2012). Evidence for Multiple Phototransduction Pathways in a Reef-Building Coral. PLoS One 7: e50371.

PARKER G H (1908). The sensory reactions of amphioxus. Proc Am Acad Arts Sci 43: 415-455.

PEI J, KIM B H, GRISHIN N V (2008). PROMALS3D: a tool for multiple protein sequence and structure alignments. Nucleic Acids Res 36: 2295-2300.

PEIRSON S N, HALFORD S, FOSTER R G (2009). The evolution of irradiance detection: melanopsin and the non-visual opsins. Phil Trans $R$ Soc B 364: 2849-2865.

PLACHETZKI D C, DEGNAN B M, OAKLEY TH (2007). The origins of novel protein interactions during animal opsin evolution. PLoS One 2: e1054.

PORATH-KRAUSE A J, PAIRETT A N, FAGGIONATO D, BIRLA B S, SANKAR K, SERB J M (2016). Structural differences and differential expression among rhabdomeric opsins reveal functional change after gene duplication in the bay scallop, Argopecten irradians (Pectinidae). BMC Evol Biol 16: 250.

PORTER M L, BLASIC J R, BOK M J, CAMERON E G, PRINGLE T, CRONIN T W, ROBINSON P R (2012). Shedding new light on opsin evolution. Proc Biol Sci 279: 3-14.

POSS S G, BOSCHUNG H T (1996). Lancelets (Cephalochordata: Branchiostomatidae): How many species are valid? Isr J Zool 42: S13-S66.
RAMIREZ M D, PAIRETT A N, PANKEY M S, SERB J M, SPEISER D I, SWAFFORD A J, OAKLEY T H (2016). The last common ancestor of most bilaterian animals possessed at least 9 opsins. Genome Biol Evol 8: 3640-3652.

RIVERAA S, OZTURK N, FAHEY B, PLACHETZKI D C, DEGNAN B M, SANCAR A OAKLEY TH (2012). Blue-light-receptive cryptochrome is expressed in a sponge eye lacking neurons and opsin. J Exp Biol 215: 1278-1286.

ROGOZINIB, MILANESI L (1997). Analysis of donor splice sites in different eukaryotic organisms. J Mol Evol 45: 50-59.

SHICHIDAY, MATSUYAMAT (2009). Evolution of opsins and phototransduction. Phil Trans $R$ Soc $B$ 364: 2881-2895.

SUGA H, SCHMID V, GEHRING W J (2008). Evolution and functional diversity of jellyfish opsins. Curr Biol 18: 51-55.

TERAKITAA (2005). The opsins. Genome Biol 6: 213.

TERAKITAA, KOYANAGI M, TSUKAMOTO H, YAMASHITA T, MIYATA T, SHICHIDA $Y$ (2004). Counterion displacement in the molecular evolution of the rhodopsin family. Nat Struct Mol Biol 11: 284-289.

TSIRIGOS K D, PETERS C, SHU N, KALL L, ELOFSSON A (2015). The TOPCONS web server for consensus prediction of membrane protein topology and signal peptides. Nucleic Acids Res 43: W401-W407.

VOPALENSKY P, PERGNER J, LIEGERTOVAM, BENITO-GUTIERREZE, ARENDT $D$, KOZMIK Z (2012). Molecular analysis of the amphioxus frontal eye unravels the evolutionary origin of the retina and pigment cells of the vertebrate eye. Proc Natl Acad Sci USA 109: 15383-15388.

WANG J K, MCDOWELL J H, HARGRAVE P A (1980). Site of attachment of 11-cisretinal in bovine rhodopsin. Biochemistry 19: 5111-5117.

YARFITZ S, HURLEY J B (1994). Transduction mechanisms of vertebrate and invertebrate photoreceptors. J Biol Chem 269: 14329-14332. 


\section{Further Related Reading, published previously in the Int. J. Dev. Biol.}

From the American to the European amphioxus: towards experimental Evo-Devo at the origin of chordates Jordi Garcia-Fernàndez, Senda Jiménez-Delgado, Juan Pascual-Anaya, Ignacio Maeso, Manuel Irimia, Carolina Minguillón, Èlia BenitoGutiérrez, Josep Gardenyes, Stéphanie Bertrand and Salvatore D’Aniello

Int. J. Dev. Biol. (2009) 53: 1359-1366

https://doi.org/10.1387/ijdb.072436jg

Evolution of CUT class homeobox genes: insights from the genome of the amphioxus, Branchiostoma floridae Naohito Takatori and Hidetoshi Saiga

Int. J. Dev. Biol. (2008) 52: 969-977

https://doi.org/10.1387/ijdb.072541nt

Peter Holland, homeobox genes and the developmental basis of animal diversity

Sebastian M. Shimeld

Int. J. Dev. Biol. (2008) 52: 3-7

https://doi.org/10.1387/ijdb.072394ss

Developmental expression of the High Mobility Group B gene in the amphioxus, Branchiostoma belcheri tsingtauense

Xiangwei Huang, Lifeng Wang and Hongwei Zhang

Int. J. Dev. Biol. (2005) 49: 49-46

http://www.intjdevbiol.com/web/paper/041915xh

Cell morphology in amphioxus nerve cord may reflect the time course of cell differentiation T C Lacalli

Int. J. Dev. Biol. (2000) 44: 903-906

http://www.intjdevbiol.com/web/paper/11206331

Embryonic development of heads, skeletons and amphioxus: Edwin S. Goodrich revisited P W Holland

Int. J. Dev. Biol. (2000) 44: 29-34

http://www.intjdevbiol.com/web/paper/10761843

Amphioxus Hox genes: insights into evolution and development

$\mathrm{J}$ Garcia-Fernàndez and $\mathrm{P}$ W Holland

Int. J. Dev. Biol. (1996) 40: S71-S72

http://www.intjdevbiol.com/web/paper/9087701

5 yr ISI Impact Factor $(2013)=2.879$

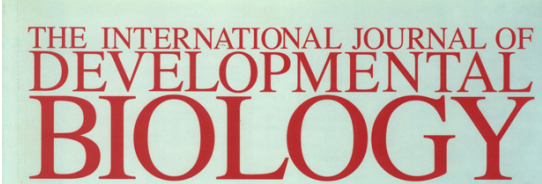

Volume 40 No. 4 August 1996

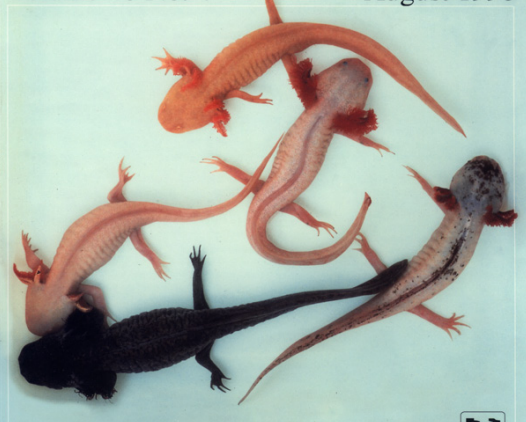

Developmental Biology of Urodeles

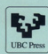

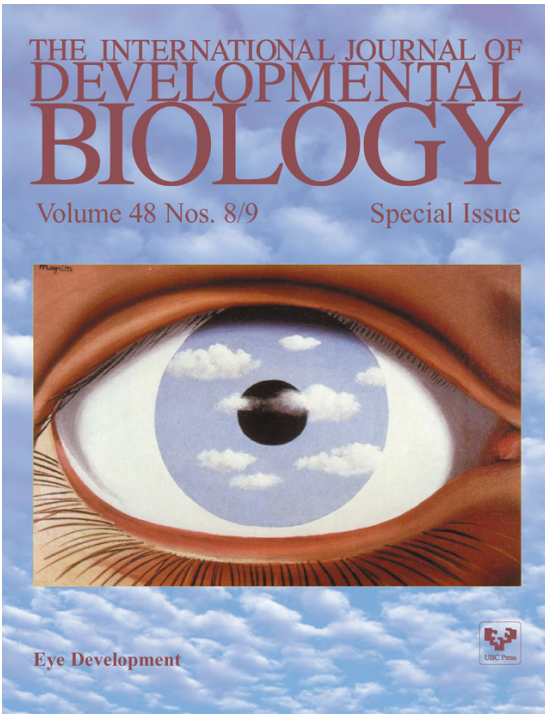

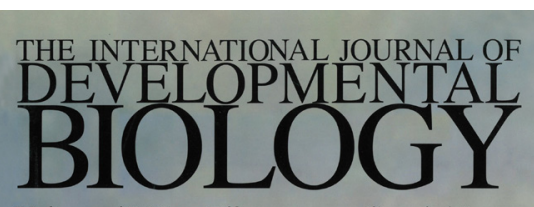

Volume 47 Nos. 7/8 Special Issue

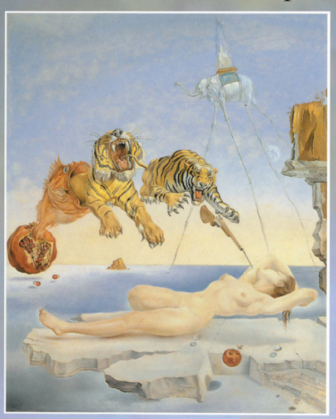

Evolution \& Development

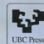

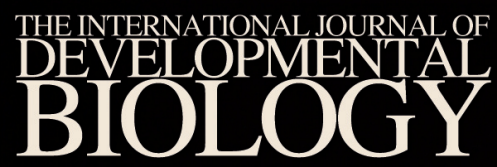

Volume 61 Nos. 10/11/12

Special Issue

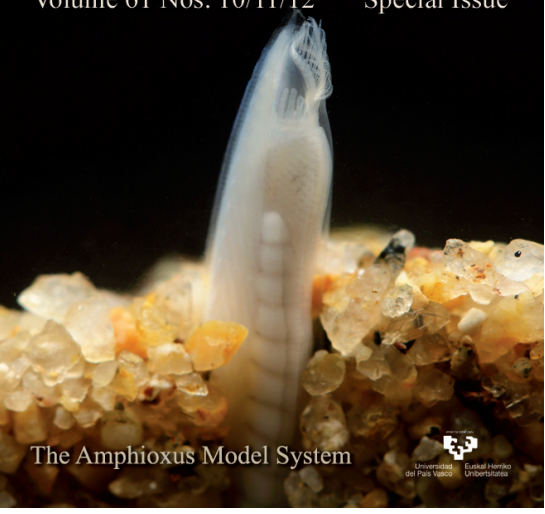

